\title{
LOS ESTILOS DE VIDA Y LOS VALORES INTERPERSONALES SEGÚN LA PERSONALIDAD EN JÓVENES DEL DEPARTAMENTO DE HUÁNUCO
}

\author{
LILIA Campos C. ${ }^{1}$ \\ Universidad Nacional Hermilio Valdizán de Huánuco, Perú \\ (RECIBIDO EL 27/10/2009, ACEPTADO EL 2/12/ 2009)
}

\begin{abstract}
RESUMEN
El objetivo del estudio es determinar la medida en que los estilos de vida y los valores interpersonales difieren según la categoría en la que se ubican dentro de cada factor de personalidad de Caprara y Barbaranelli en los jóvenes del departamento de Huánuco.

El método fue descriptivo correlacional, la muestra conformada por 374 jóvenes de ambos sexos a quienes se les aplicó: el Cuestionario BFQ "Big Five", el Cuestionario de Valores Interpersonales "SIV" y el Cuestionario de Estilos de Vida. La validez y confiabilidad de dichos instrumentos fueron debidamente demostrados adaptando a baremos peruanos. Los resultados evidencian que los factores de personalidad del Big Five, no plantean diferencias significativas sobre los estilos de vida y los valores interpersonales.
\end{abstract}

Palabras clave: personalidad, estilos de vida, valores interpersonales.

\section{ABSTRACT \\ LIFE STYLE AND INTERPERSONAL VALUES ACCORDING TO YOUNG PEOPLE PERSONALITY FROM HUANUCO DEPARTAMENT}

The work has the the objective of determining how way the life style and interpersonal values differ each other accordint to tha category that each youg people from Huanuco Department obtains into from each personality factor of Caprara and Barbaranelli.

The used method was the Correlational Descriptive, the sample was conformed for 374 both sex young people and responded the next tests: BFQ Questionary, the Interpersonal Values Questionary and the Life Style Questionary. The Validity and Trusting of these tests were shown and adapted to peruvian scales.

The results show that personality factors from Big Five don't set out significative difference over life styles and interpersonal values.

Keywords: Personality, life styles, interpersonal values.

1 Docente principal de la Facultad de Medicina Humana y Psicología de la UNHEVAL, Huánuco. 


\section{INTRODUCCIÓN}

El mundo actual, caracterizado por los cambios acelerados que experimenta, ha producido modificaciones en todos los terrenos, particularmente en el mundo subjetivo del hombre, lo que comprende la dimensión valorativa y actitudinal donde los jóvenes son más sensibles a estos cambios. Por otro lado, la vida social y las relaciones interpersonales están seriamente afectadas, no solo por los estragos de la pobreza sino por una crisis moral que afecta a la sociedad en su conjunto y en todos los niveles, en nuestro caso particular, nos interesa aproximarnos a los jóvenes de educación superior porque son el futuro de la intelectualidad y la participación futura en el desarrollo de nuestra región y país, y porque hay la necesidad de formar profesionales que no solo destaquen en conocimientos relacionados con su especialidad, sino también que sean personas íntegras y ciudadanos comprometidos con la cultura, la historia y el bienestar de nuestra sociedad.

Krauskopf (2006) señala que en las décadas recientes se han hecho muchos avances en el campo de la salud y en la atención a la situación de la juventud. La prolongación de la esperanza de vida, la modernidad y la globalización con su gran gama de complejos estímulos, incrementa la necesidad de los adolescentes y jóvenes de encontrar, en las nuevas circunstancias que les rodean, los elementos para asumir los cambios biopsicosociales que experimentan en la construcción de un rol social que coincida con la identidad, organizar su comportamiento desde un hacer en el mundo que los objetive positivamente y sustente un proyecto de vida que dé sentido a su relación presente con el entorno, lo que en muchas áreas de América Latina incluye, además, las dificultades propias de la pobreza.

En la etapa juvenil se consolida notablemente la personalidad y las relaciones sociales del individuo. En diferentes ocasiones se ha afirmado (Espinoza 2001, Ochaita, 2003) que los progresos en el desarrollo cognoscitivo y afectivo de los jóvenes le conducen a exigir que sea reconocida su creciente necesidad de autonomía, su capacidad de participación y toma de decisiones en los ámbitos familiar, educativo y social, en la que los adultos perciben numerosos peligros y riesgos, es decir, una etapa de la vida en que la indefinición del estatus del sujeto le coloca en un estadio decisivo para su futuro como adulto, porque en esta etapa se va dilucidar el resto de su vida. Pero, a la vez, las características de personalidad del joven no pueden considerarse universales dado que no derivan exclusivamente de lo biológico, sino además del propio individuo en desarrollo, dentro del contexto social en el que dicha personalidad se desarrolla.

Sin embargo, en la actualidad nos encontramos con valores y creencias culturales muy diferentes a los tiempos anteriores, del grado en que éstos encuentren expresión en la vida diaria depende, en gran parte, la satisfacción personal.

Leonard Gordon (1979) refiere que los valores interpersonales constituyen aquellos medios que determinan lo que las personas hacen y cómo lo hacen y que éstas están influidas conscientes o inconscientemente por el sistema de valores que ellos adopten. Asimismo, los valores interpersonales constituyen la compatibilidad e incompatibilidad entre valores inter o intraindividuales; es decir, aquel que implica relaciones de un individuo con los demás.

Por otro lado, se pretende estudiar los estilos de vida de los jóvenes lo cual se va conformando a lo largo de su historia del sujeto, es un proceso de aprendizaje, ya sea por asimilación, imitación o adaptación de modelos, de patrones familiares o grupos informales. 
Durante las últimas dos décadas, los cambios en los patrones de participación educativa, la larga transición al mercado laboral y una extensión (temporal-espacial) en el periodo de dependencia familiar, han tenido sus consecuencias en los estilos de vida que los jóvenes adoptan y en las maneras en las que aprovecha su tiempo libre. En la modernidad, la gente se enfrenta a un gran número de opciones en muchos aspectos de sus vidas, pero, al mismo tiempo, queda sujeta a un conjunto importante de restricciones e influencias.

Florez y Alarcón (1998) señalan que el estilo de vida no puede formarse espontáneamente, sino como decisiones conscientes de los hombres y producto de los factores personales, ambientales y sociales que convergen no solo del presente, sino de la historia interpersonal, y que al mismo tiempo puede favorecer la consolidación de algunos altos valores, y aún más, su difusión social. El estilo de vida es un aspecto de la actividad vital diaria de la persona que indica la línea y orientación de la conducta y el razonamiento de la personalidad; en él están reflejados los hábitos estables, las costumbres, los conocimientos, así como los valores, aspiraciones y también los riesgos en sus conductas; que conociendo podemos influir para optimizar el desarrollo de los jóvenes. El estilo de vida puede ayudar a consolidar los valores en relación con sus hábitos de ejercicio, alimentos que ingiere, las prácticas nutricionales adecuadas, los comportamientos de seguridad, la reducción del consumo de sustancias, desarrollo de competencias para establecer relaciones sociales, minimizar el estrés, accidentes, medio ambiente y sexualidad.

Infiesta (1994) señala que todo estilo de vida está favorecido por los hábitos, costumbres, modas, valores existentes y dominantes en cada momento en cada comunidad; estos factores tienen como característica común que son aprendidos y por tanto modificables a lo largo de toda la vida. Comas Arnau (2003) mediante un estudio ha podido identificar los estilos de vida reales de los jóvenes urbanos en España, en jóvenes de 15 a 24 años. Encontró que se produce una influencia mutua entre estilos de vida y valores y cómo a la vez determinados estilos de vida concentran riesgos particulares.

A partir de estos planteamientos, se pretende conocer mejor la realidad en la que se desarrolla la vida de los jóvenes del departamento de Huánuco. En ella queremos abordar el análisis de los comportamientos de los jóvenes que viven en el entorno urbano, poniendo en consonancia tres componentes importantes que forman parte y que se derivan de esas conductas. Por un lado, sus características de personalidad, por otro, los valores que ponen en juego y que incorporan en su interrelación social y finalmente, los distintos estilos de vida y los riesgos que corren y que se manifiestan sobre todo en los centros de estudios; para lo cual nos planteamos la siguiente interrogante:

¿En qué medida los estilos de vida y los valores interpersonales difieren según la categoría en la que se ubican dentro de cada factor de personalidad de Caprara y Barbaranelli en los jóvenes del departamento de Huánuco?

\section{MÉTODO}

El método utilizado para despejar los problemas planteados pone especial énfasis en la agrupación previa de los examinados según la categoría en la que se ubican en cada uno de los factores de personalidad de Caprara, G., Barbaranelli, C., y Borgogni, L.; para contrastar los estilos de vida y los valores interpersonales entre los grupos polarizados por 
factor de personalidad ubicando el presente estudio en el método descriptivo correlacional, diseño transversal (Hernández R. y otros, 2003).

La muestra de trabajo fue probabilística, constituida por 374 jóvenes con edades entre 18 y 25 años de las provincias de Pachitea, Leoncio Prado, Lauricocha y Ambo, del departamento de Huánuco, de ambos sexos y estudiantes de Educación Superior. Para la recolección de los datos se utilizó la técnica de la encuesta y los siguientes instrumentos:

1. El Cuestionario BFQ, "BIG FIVE", de los autores G.V. Caprara, C. Barbaranelli y L. Borgogni (1993); procede de la Organizzazioni Speciali (OS), Florencia, fue adaptado por J. Mermúdez, Catedrático de Psicología, UNED, Madrid, y pone de manifiesto la preeminencia de la solución de cinco factores respecto de otras soluciones denominados: energía, afabilidad, tesón, estabilidad emocional y apertura mental. En cuanto a la validez, los datos obtenidos, además de mostrar una elevada validez convergente de las escala BFQ, ponen de relieve su validez discriminante, corroborada por la ausencia de correlaciones elevadas con dimensiones relativas a diferentes características de la personalidad.

2. Cuestionario de Estilo de Vida: se utilizó un inventario creado en la Universidad de Berkeley por Sheldon Margen, publicado en la "Wellness Enciclopedia", adaptado al español por el médico Manuel Garcés, en Guatemala, y adaptado en Bogotá el año 1998 por Luis Flores Alarcón con las siguientes áreas: condición física, hábitos alimentarios, consumo de alcohol y otras sustancias, accidentes, valores de la vida, estrés y apoyo social, medio ambiente y sexualidad. La revisión para el presente estudio de la confiabilidad está basada en la relación subtests.

3. Cuestionario de Valores Interpersonales - SIV, cuyo nombre original es Survey of Interpersonal Values, fue elaborado por Leonard V. Gordon, Ph.D.(1962), traducido y adaptado al español por José A. Corteza y equipo de colaboradores y posteriormente adaptado y estandarizado a nuestra realidad por Leonardo Higueras (1972) para estudiantes de secundaria y superior. Este cuestionario ofrece medidas dentro de un segmento del dominio de valores, justamente de aquel que implica relaciones de un individuo con los demás, tal tipo de valores tiene relevancia en el ajuste personal, social, familiar y profesional del sujeto. Las escalas son las siguientes: Soporte, conformidad, reconocimiento, independencia, benevolencia y liderazgo.

En relación con la validez, el SIV fue desarrollado mediante el uso del análisis factorial; puede decirse, por lo tanto, que las escalas del SIV tienen validez factorial, asimismo ha sido correlacionado con otras medidas de valores y de rasgos de personalidad.

Con los datos obtenidos se elaboró la data correspondiente enfatizando en las variables demográficas, especialmente en la estructura de los ítems de los cuestionarios o los test utilizados. Luego se procedió a analizar si se distribuyen normalmente; con la prueba de Smirnov Kolmogorov, se encontraron valores $\mathrm{Z}$ cuya probabilidad (P) fueron menores de 0.05 razón por la cual podemos afirmar que los datos obtenidos no guardan una distribución normal estadística por lo que los análisis efectuados fueron con estadísticos no paramétricos, utilizando para la contrastación la prueba de U de Mann-Whitney. 


\section{RESULTADOS}

De acuerdo con el enfoque de la personalidad expresado en factores de Caprara, G., Barbaranelli, C., Borgogni, L. hemos creído analizar si es conveniente o no utilizar las normas que ellos obtuvieron en la muestra española, habiendo encontrado que en todos los factores, peruano y españoles, difieren significativamente, dejando entrever que los factores son influenciados socioculturalmente, por tanto, es posible que algunos de los componentes medidos resulten productos de aprendizajes más que de razones tipo rasgo.

Análisis comparativo de los estilos de vida según las categorías en cada factor de la personalidad

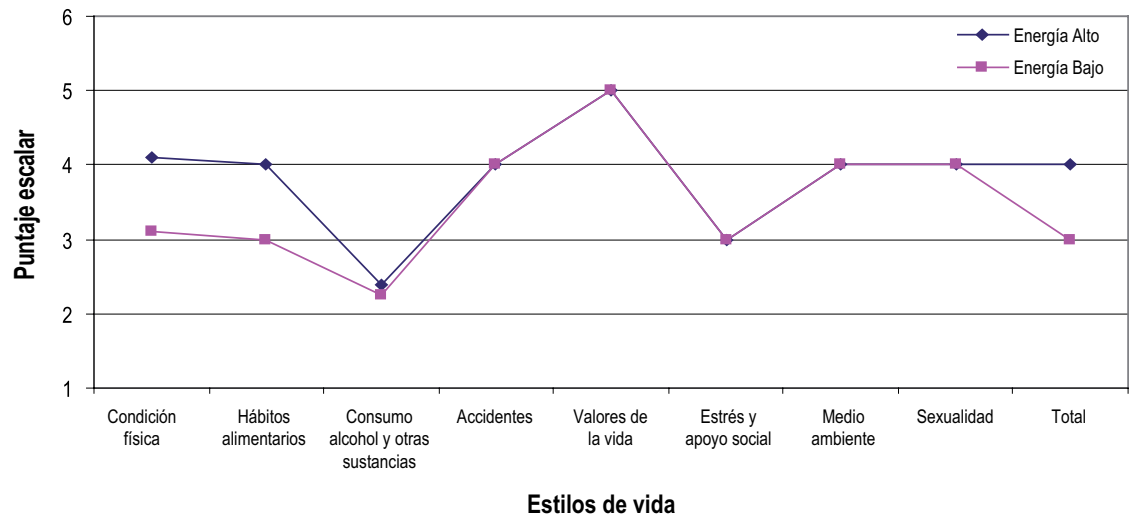

Figura N.$^{0}$ 1. Estilos de vida según la alta y baja puntuación en el factor Energía de la personalidad, en jóvenes de la ciudad de Huánuco.

De la figura se desprende que el estilo de vida ubicado en la categoría superior corresponde a valores de vida en relación con la espiritualidad e intelectualidad y se abstienen del consumo de alcohol y otras sustancias.

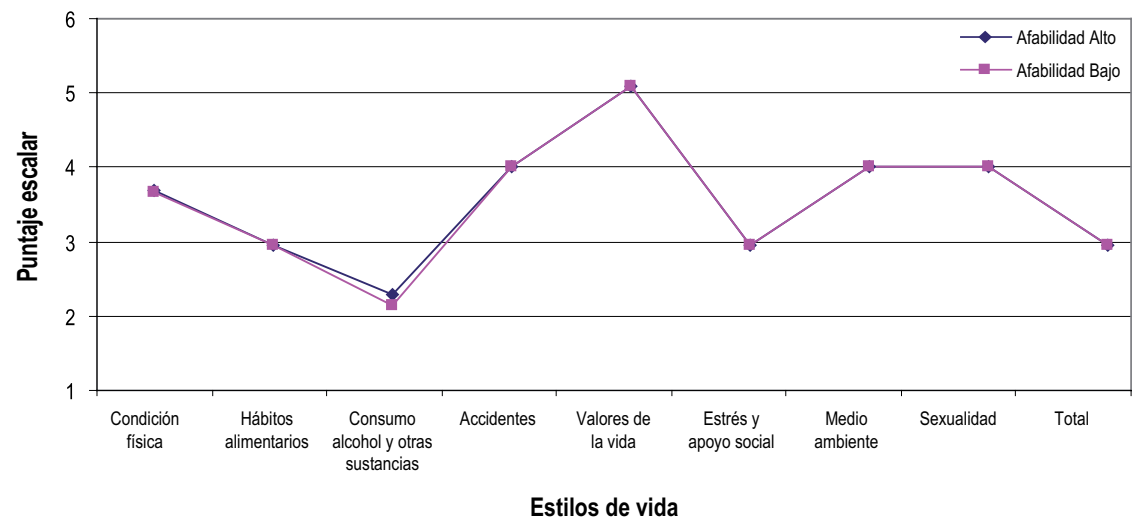

Figura N. ${ }^{\circ}$ 2. Estilos de vida según la alta y baja puntuación en el factor Afabilidad de la personalidad, en jóvenes de la ciudad de Huánuco. 
La figura encontrada esta vez en relación al factor afabilidad de la personalidad nos arroja resultados similares, donde la mayor puntuación corresponde a valores de vida y la menor a conducta protectora es para el consumo de alcohol y otras sustancias.

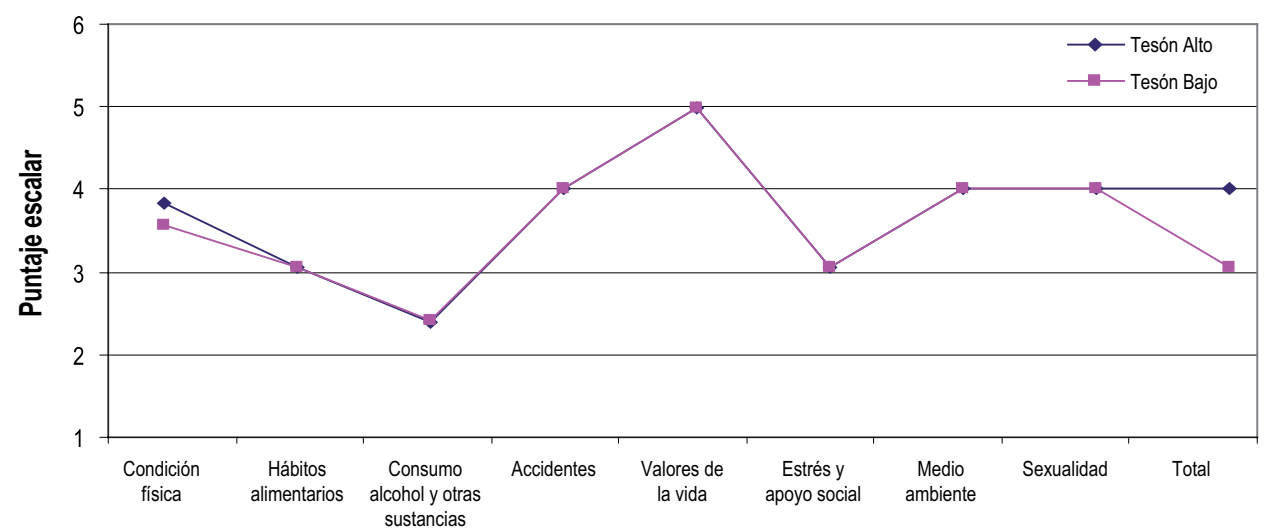

Estilos de vida

Figura N. ${ }^{\circ}$ 3. Estilos de vida según la alta y baja puntuación en el factor Tesón de la personalidad, en jóvenes de la ciudad de Huánuco.

La similitud de las funciones de los estilos de vida en función a la alta y baja puntuación en el factor tesón con el factor afabilidad, refuerza lo encontrado, estos factores son independientes de los estilos de vida, no interesa cuan escrupuloso detallista y perseverante resulte una persona para que sus estilo de vida sea parecido en cualquiera de los extremos de este factor de personalidad.

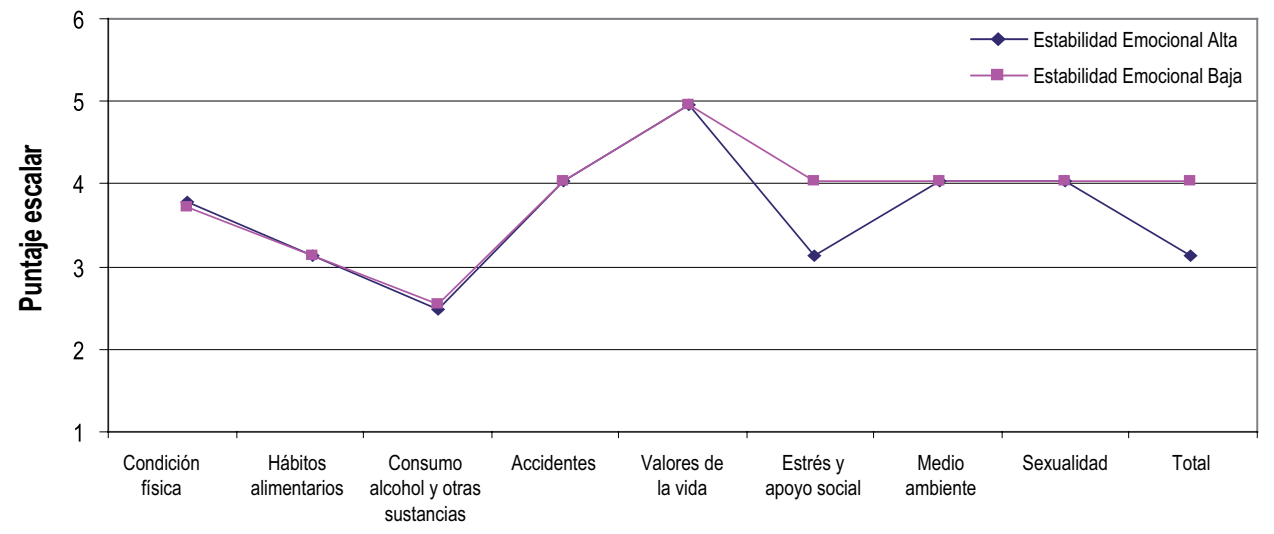

Estilos de vida

Figura N. ${ }^{\circ}$ 4. Estilos de vida según la alta y baja puntuación en el factor Estabilidad Emocional de la personalidad, en jóvenes de la ciudad de Huánuco. 
De la figura se observa que el único cambio significativo en relación a las anteriores corresponde al estilo de vida: Estrés y apoyo social, donde la puntuación más baja corresponde a los jóvenes que tienen baja estabilidad emocional como factor de personalidad.

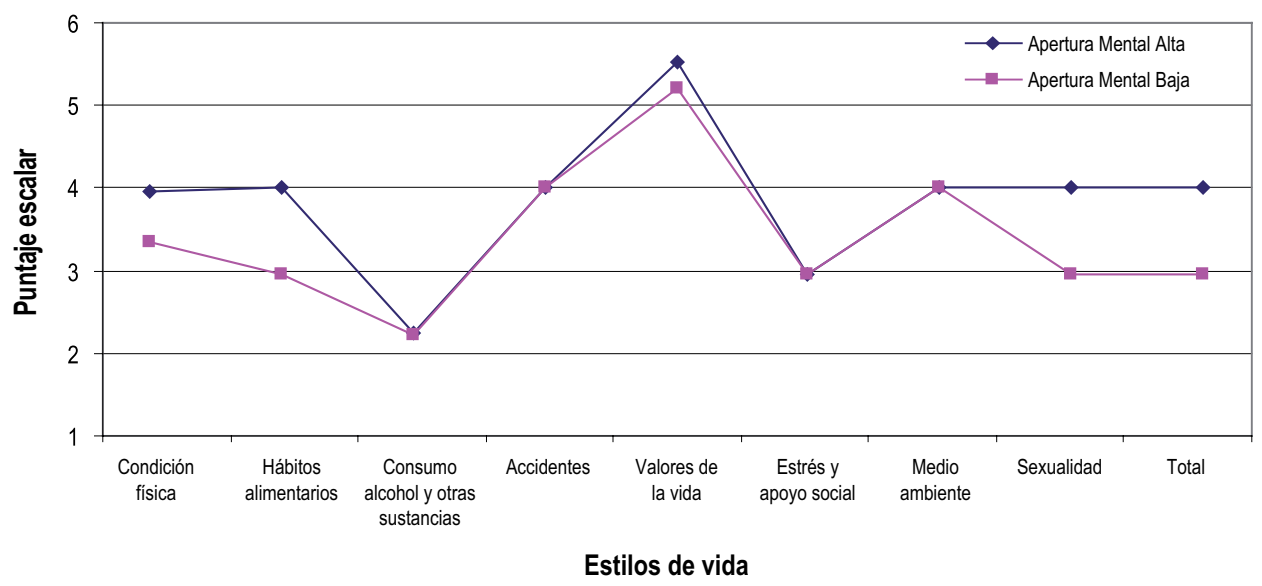

Figura $\mathbf{N}^{0}{ }^{5}$. Estilos de vida según la alta y baja puntuación en el factor Apertura Mental de la personalidad, en jóvenes de la ciudad de Huánuco.

Los jóvenes con apertura mental alta tienen como estilos de vida condiciones físicas diferenciadas lo mismo ocurre en los estilos de vida correspondiente a hábitos alimentarios, valores de vida y sexualidad.

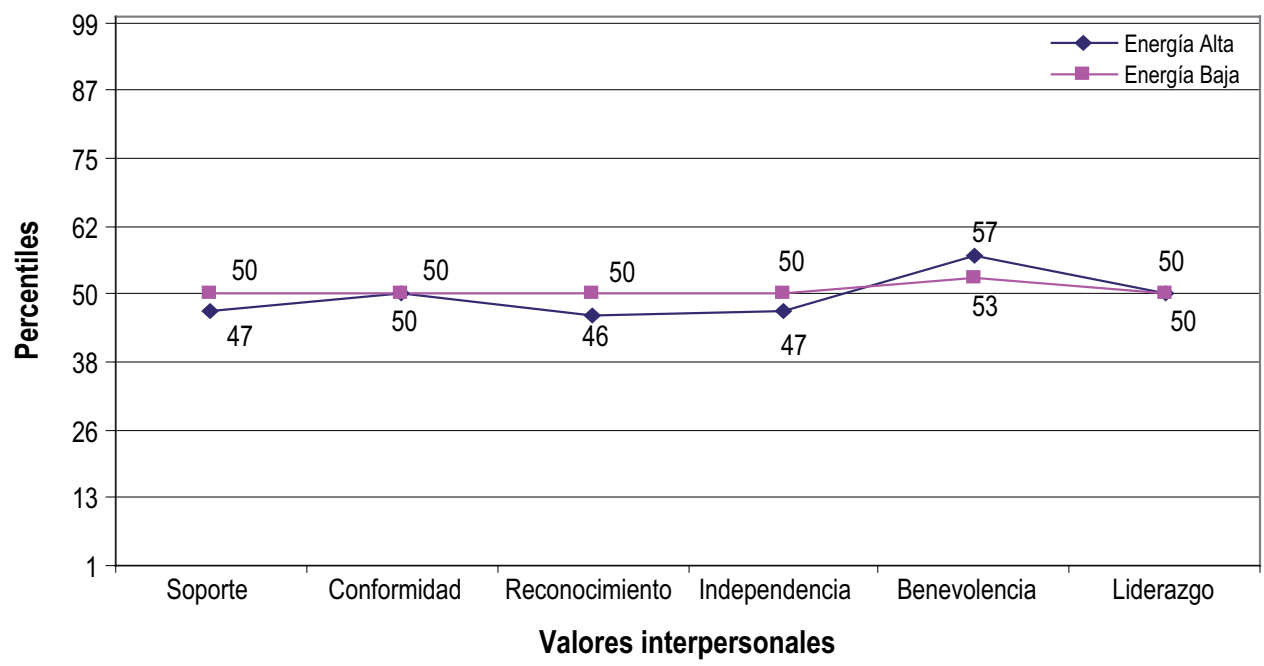

Figura N. ${ }^{\circ}$ 6. Valores interpersonales según la alta y baja puntuación en el factor Energía de la personalidad, en jóvenes de la ciudad de Huánuco. 
De la figura se puede ver que el factor energía sólo plantea diferencias en los valores interpersonales mencionados.

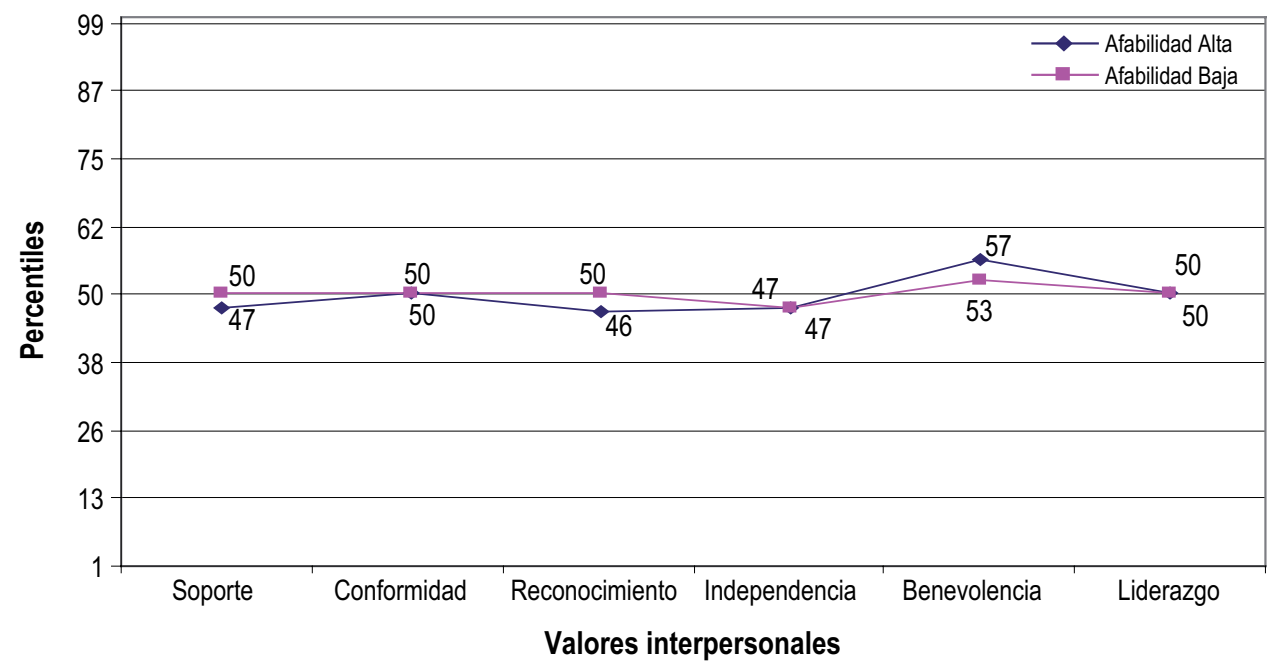

Figura N. ${ }^{\circ}$ 7. Valores interpersonales según la alta y baja puntuación en el factor Afabilidad de la personalidad, en jóvenes de la ciudad de Huánuco.

De la figura se desprende la similitud en los valores interpersonales independientemente de la alta o baja afabilidad como factor de la personalidad, con excepción del valor interpersonal de benevolencia.

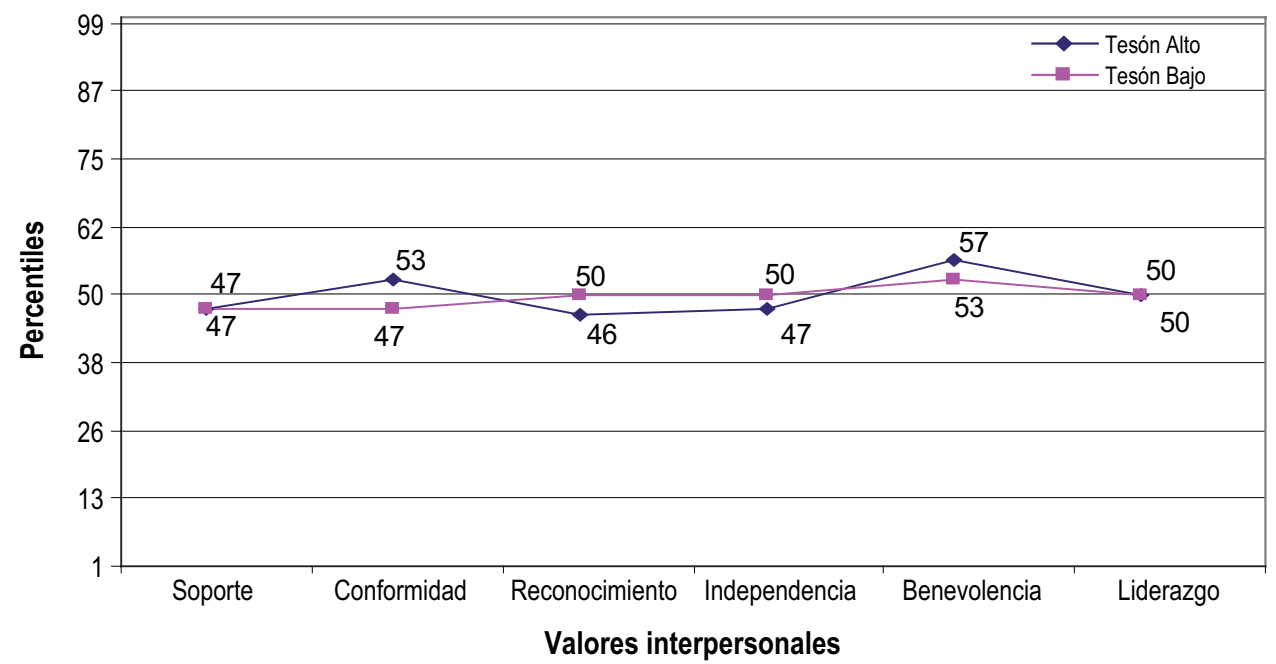

Figura $\mathbf{N}^{\mathbf{0}} \mathbf{8}$. Valores interpersonales según la alta y baja puntuación en el factor Tesón de la personalidad, en jóvenes de la ciudad de Huánuco. 
La figura nos permite visualizar lo descrito líneas arriba, los jóvenes con alto tesón presentan mayor conformismo menor deseo de reconocimiento y mayor entrega a las necesidades del prójimo.

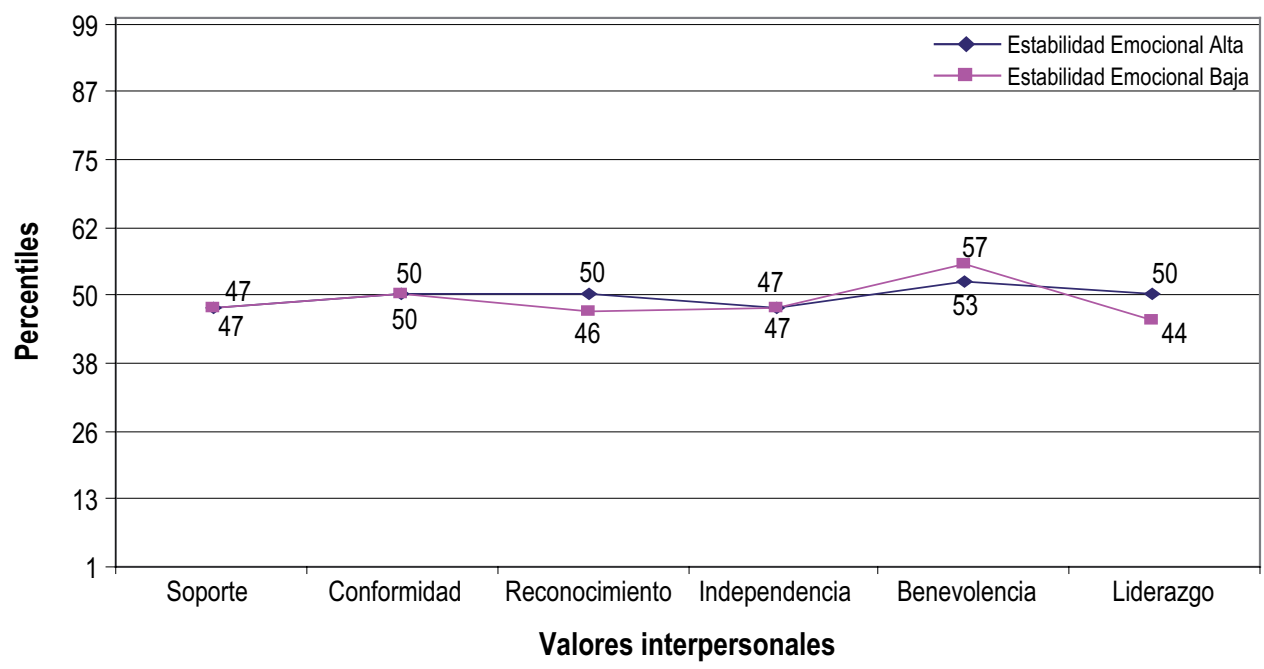

Figura $\mathbf{N}^{0}$ 9. Valores interpersonales según la alta y baja puntuación en el factor Estabilidad Emocional de la personalidad.

En la figura podemos leer la similitud de los resultados alrededor del percentil 50, sin visualizar variaciones significativas.

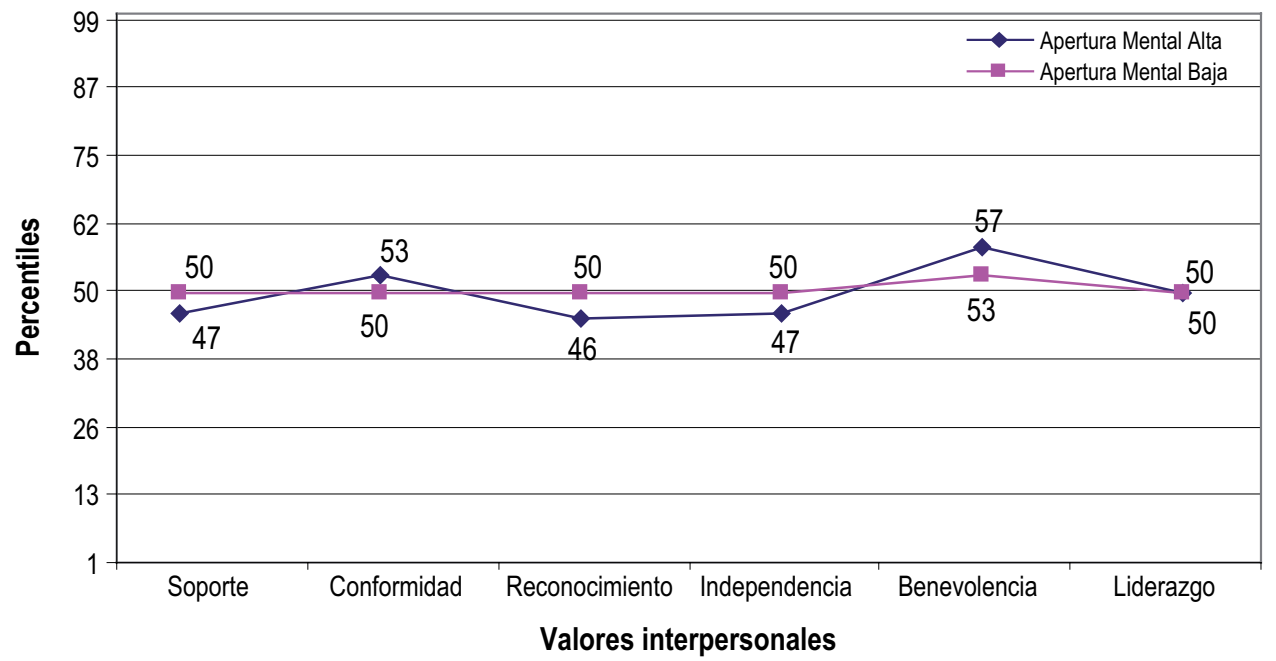

Figura $\mathbf{N}^{0}$ 10. Valores interpersonales según la alta y baja puntuación en el factor Apertura Mental de la personalidad, en jóvenes de la ciudad de Huánuco. 
La figura confirma los resultados descritos líneas arriba, mayor reconocimiento de los jóvenes con baja apertura mental, mayor benevolencia en los jóvenes con alta apertura mental.

\section{DISCUSIÓN}

En nuestra investigación para explicar la personalidad hemos cogido el modelo Big Five o Cinco Grandes Factores que propone como un intento de mediación y unificación entre los distintos puntos de vista y donde convergen dos tradiciones de investigación diferentes, la lexicográfica y la factorialista, y que los resultados difieren con la muestra española ya que en la muestra peruana no hay diferencias por sexo; sin embargo, sí existen diferencias por sexo en la muestras española; por lo que se ha establecido el baremo peruano.

En cuanto a la variable Estilos de Vida relacionada con los factores de personalidad se acepta la hipótesis nula, por lo que no establecen diferencias significativas, sabemos que los estilos de vida se van conformando en el proceso de desarrollo del individuo como un producto complejo de factores personales, ambientales y sociales como señala (Bibeau, 1985) y que comprende tres aspectos interrelacionados: lo material que se caracteriza por manifestaciones de la cultura material, lo social según las formas y estructuras organizativas y en el plano ideológico se expresan a través de ideas, valores y creencias. En tal sentido las características de los estilos de vida de los jóvenes del departamento de Huánuco no están aislados del contexto social, económico, político y cultural, puesto que los jóvenes procedentes de las diferentes provincias con sus características peculiares, emigran a la ciudad en búsqueda de mejorar su calidad de vida ya sea por estudios y/o trabajo y donde van asimilando modelos y comportamientos de formas de vida específica que reflejan las actitudes, los valores o la visión del mundo que tienen los jóvenes.

Teniendo en cuenta las características de la juventud y los factores que influyen en la formación de su personalidad, los jóvenes son vulnerables a las situaciones de riesgo y está relacionado con los estilos de vida. Weinstein (1992) señala que cuando las bases del desarrollo de la identidad son deficitarias, su afirmación se establece carente de un compromiso estructurante que busque evitar los peligros para poder preservar los logros presentes y futuros, incrementándose las conductas de riesgo en búsqueda de la satisfacción inmediata o la autoafirmación con acciones efímeras que dan sensaciones de logro y reconocimiento.

Sin embargo, esto es un aspecto que no se considera en la educación, así Puelles, M. (2000) nos revela que la problemática del estilo de vida no ha sido planteada como un objeto de investigación o de intervención educativa desde los sistemas escolares.

Con relación a nuestra hipótesis sobre la relación de los factores de personalidad con los valores interpersonales, hemos encontrado diferencias significativas en reconocimiento y benevolencia.

Los jóvenes con alta energía presentan poca necesidad de ser elogiados y reconocidos, igual con relación a benevolencia experimentan mayor sensibilidad social y amor al prójimo que los jóvenes con baja energía. González, O. (1976) señala que en los jóvenes toda la personalidad sufre una transformación significativa bajo la influencia del 
marcado desarrollo de las esferas motivacional e intelectual, se da un proceso intensivo de asimilación de las reglas sociales que sustenta el grupo en el cual se desenvuelven, asumen una posición crítica a partir de elaboraciones propias y convicciones y una de sus manifestaciones son su sensibilidad social, de solidaridad con los demás, se muestran muy activos, dinámicos y locuaces y la Universidad es un espacio que les permite desarrollar estos rasgos y valores. Así los investigadores M. Crispín, S. Noguez y H. Patiño (1998) consideran que el desarrollo del juicio moral y los valores en los estudiantes universitarios se produce de acuerdo con un orden lógico (enfoque cognitivo) en el que las estructuras formales poseen carácter universal, aunque en cuanto a su contenido es relativo a cada cultura. Teresa Sanz (1996) plantea que los jóvenes se caracterizan por un mayor caudal de conocimientos, de experiencias propias más profundas y por una mayor capacidad para el pensamiento abstracto y generalizador, lo que permite comprender el contenido esencial de las normas sociales generales, aunque el desarrollo intelectual que el joven alcanza depende fundamentalmente del tipo de enseñanza recibida. Esto implica también las pautas y normas establecidas en su contexto no solo educativo sino también familiar y social.

Los valores tienen un carácter supraindividual que tiene significación en toda la sociedad y que el valor puede quedar reflejado en una o varias cualidades de la personalidad de los individuos; como señala A. Delgado (1998) que aunque los valores poseen un contexto histórico y cultural en el cual se desarrollan, existe cierto consenso que consideran que los valores deseables en los alumnos universitarios son, entre otros, la responsabilidad, la libertad y la independencia.

\section{CONCLUSIONES}

1. Habiendo efectuado cuarenta contrastaciones que resultan de comparar a los examinados ubicados en cada una de las categorías alta y baja de los cinco factores de personalidad de Big Five, en función a ocho estilos de vida, hemos encontrado que sólo en ocho contrastaciones, que representa el $20 \%$, hay diferencias significativas, en tanto que en el $80 \%$ no hay diferencias, razón por la cual estamos en condiciones de expresar lo siguiente:

1.1. Se afirma que los factores de la personalidad del Big Five no plantean diferencias significativas sobre los estilos de vida.

1.2. Los contenidos de los factores de personalidad al parecer se refieren a factores comportamentales adquiridos antes que a factores tipo rasgo de personalidad.

2. Habiendo efectuado treinta contrastaciones que resultan de comparar a los examinados ubicados en cada una de las categorías alta y baja de los cinco factores de personalidad de Big Five, en función a seis valores interpersonales de Gordon, hemos encontrado que sólo en el 30\% hay diferencias significativas, en tanto que en el $70 \%$ no las hay, razón por la cual estamos en condiciones de afirmar:

2.1. Se acepta la hipótesis nula que afirma que los factores de la personalidad del Big Five no plantean diferencias significativas sobre los valores interpersonales. 
2.2. Dado que los valores interpersonales no están determinados por los factores de la personalidad, que al no encontrar determinantemente diferencias significativas implica que los componentes de los valores interpersonales son de naturaleza sociocultural productos del aprendizaje, posiblemente similar a lo evaluado por el Big Five.

\section{REFERENCIAS BIBLIOGRÁFICAS}

1. Barbaranelli y Caprara (1996). Cuestionario de Personalidad BFQ.

2. Comas, D. (2003). Jóvenes y estilos de vida. España.

3. Costa y McCrae (1998). Joint factors in self reports and ratings, neuroticismo, extraversión and openess to experience. Personality and Individual Differences. Greenwich,CT: JAI Press.

4. Crispín, M.; García, L.; Noguez, S. y Patiño, H. (1998). El desarrollo del juicio moral en los estudiantes universitarios, Revista Didáctica (38) pp. 13-16, México, D.F.: Universidad Iberoamericana.

5. Delgado, A. (1998). Estudio de las relaciones entre la necesidad cognitiva y las dimensiones de la personalidad: extraversión, emocionalidad y dureza en estudiantes universitarios. Revista de Investigación en Psicología. UNMSM, Vol. III (3), pp. 131-143.

6. Espinoza (2001). Desarrollo de la juventud, Colombia.

7. Flores y Alarcón (1998). Estilos de vida en jóvenes de Chiapas. Colombia.

8. González. O. (2000). La educación moral. Comunicación, personalidad y desarrollo, pp. 100 -111. La Habana: Ed. Pueblo y Educación.

9. Gordon, L. (1979). Inventario de valores interpersonales SIV.

10. Infiesta J.A.; Garrucho G. (1994). Sevilla. Estilos de Vida. Abordaje desde el nivel local.

11. Puelles M. (2000). Revista de educación, pp. 7-36.

12. Sanz, T. (1996). Características psicológicas del período juvenil. La Habana: CEPES.

13. Krauskopf, D. y Cabezas, M. (1992). Condiciones psicosociales del embarazo en adolescentes. Actualidades en psicología, Vol. 5, N. ${ }^{\circ}$ 42. Instituto de Investigaciones Psicológicas de la Universidad de Costa Rica. San José.

14. Weinstein, J. (1992). Riesgo psicosocial en jóvenes. Santiago de Chile: PREALC. 